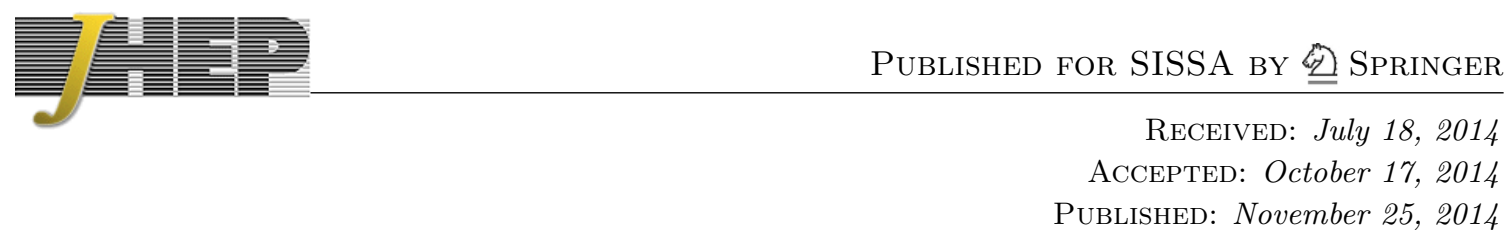

\title{
Higgs mixing in the NMSSM and light Higgsinos
}

\author{
Kwang Sik Jeong, ${ }^{a, b}$ Yutaro Shoji ${ }^{c}$ and Masahiro Yamaguchi ${ }^{c}$ \\ ${ }^{a} D E S Y$, \\ Notkestrasse 85, 22607 Hamburg, Germany \\ ${ }^{b}$ Center for Theoretical Physics of the Universe, \\ IBS, Daejeon 305-811, Korea \\ ${ }^{c}$ Department of Physics, Tohoku University, \\ Sendai 980-8578, Japan \\ E-mail: ksjeong@ibs.re.kr, yshoji@tuhep.phys.tohoku.ac.jp, \\ yama@tuhep.phys.tohoku.ac.jp
}

ABSTRACT: We explore the effects of Higgs mixing in the general next-to-minimal supersymmetric Standard Model (NMSSM). Extended to include a gauge singlet, the Higgs sector can naturally explain the observed Higgs boson mass in $\mathrm{TeV}$ scale supersymmetry without invoking large stop mixing. This is particularly the case when the singlet scalar is light so that singlet-doublet mixing increases the mass of the SM-like Higgs boson. In such a case the Higgs mixing has interesting implications following from the fact that the higgsino mass parameter and the singlet coupling to Higgs bilinear crucially depend on the Higgs boson masses and mixing angles. For the mixing compatible with the current LHC data on the Higgs signal rates, the higgsinos are required to be relatively light, around or below a few hundred $\mathrm{GeV}$, as long as the heavy doublet Higgs boson has a mass smaller than about $250 \sqrt{\tan \beta} \mathrm{GeV}$ and the singlet-like Higgs boson is consistent with the LEP constraint. In addition, the Higgs coupling to photons can receive a sizable contribution of either sign from the charged-higgsino loops combined with singlet-doublet mixing.

Keywords: Supersymmetry Phenomenology

ARXIV EPRINT: 1407.0955 


\section{Contents}

1 Introduction 1

2 Higgs properties in the NMSSM 2

2.1 Higgs sector 2

2.2 SM-like Higgs boson 4

$\begin{array}{lll}3 & \text { NMSSM with a light singlet scalar } & 6\end{array}$

3.1 LHC constraints on Higgs mixing $\quad 7$

$\begin{array}{ll}3.2 & \text { Implication of Higgs mixing on higgsino properties }\end{array}$

$\begin{array}{lll}3.3 & \text { CP-odd Higgs bosons } & 14\end{array}$

$\begin{array}{lll}3.4 & \text { Neutralino sector } & 15\end{array}$

$\begin{array}{lll}4 & \text { Conclusions } & 17\end{array}$

\section{Introduction}

Supersymmetry (SUSY) provides a natural solution to the gauge hierarchy problem of the Standard Model (SM), and is a leading candidate for new physics at the TeV scale $[1,2]$. To be consistent with the LHC data, the supersymmetric extension of the SM should accommodate a scalar boson that has a mass near $126 \mathrm{GeV}$ and behaves like the SM Higgs boson [3, 4]. The minimal supersymmetric SM (MSSM) can explain the observed mass if one considers heavy stops above $7 \mathrm{TeV}$ or large stop mixing, which however would cause severe fine-tuning in the electroweak symmetry breaking. Such a difficulty can be avoided in the next-to-minimal supersymmetric SM (NMSSM) [5-12], where the Higgs sector is extended to include a gauge singlet $S$ interacting with the Higgs doublets via the superpotential coupling, $\lambda S H_{u} H_{d}$. The NMSSM can provide a larger mass to the SM-like Higgs boson because there are additional tree-level contributions from the $F$-term scalar potential $\lambda^{2}\left|H_{u} H_{d}\right|^{2}$, and from the singlet-doublet mixing.

The Higgs sector has a rich structure in the NMSSM due to the singlet scalar. In particular, singlet-doublet scalar mixing can have interesting phenomenological consequences [1331]. It increases the mass of the SM-like Higgs boson if the singlet scalar is light, which does not require low $\tan \beta$ or sizable $\lambda$ differently from the singlet $F$-term contribution. In addition, the singlet-doublet mixing induces a Higgs coupling to photons of either sign through the charged-higgsino loops $[13,14]$. In this paper we study the effects of Higgs mixing in the NMSSM where the lightest CP-even neutral scalar is singlet-like, i.e. lighter than the SM-like Higgs boson $h$, under the assumption that all the superparticles have masses around or below $\mathrm{TeV}$ as would be required to solve the hierarchy problem. Since 
our results apply to any NMSSM model, we will not specify the exact form of the singlet superpotential or the mediation mechanism of SUSY breaking.

In the presence of scalar mixing, $h$ has properties deviated from those of the SM Higgs boson. However sizable mixing is still compatible with the current experimental data on the Higgs signal rates in TeV scale SUSY, especially when the singlet-doublet mixing increases the mass of $h$. The scalar mixing depends on the coupling $\lambda$ and the higgsino mass parameter $\mu$. This implies that the LHC and LEP constraints on scalar mixing are converted into the constraints on $\lambda$ and $\mu$, and vice versa. Interestingly it turns out that higgsinos are required to be light as $h$ becomes more SM-like in NMSSM models where the singlet-like Higgs boson is lighter than $h$. We find that higgsinos have masses around or below a few hundred $\mathrm{GeV}$ for the scalar mixing compatible with the LHC results on Higgs signal rates, as long as the heavy doublet Higgs boson has a mass less than about $250 \sqrt{\tan \beta} \mathrm{GeV}$. The upper bound on $\mu$ also indicates that the heavy doublet Higgs boson should have a large mass at large $\tan \beta$ in order to allow $|\mu|>100 \mathrm{GeV}$ in the viable region of scalar mixing, as suggested by the LEP bound on the chargino mass [32].

This paper is organized as follows. In section 2 we discuss some generic features of scalar mixing in the NMSSM Higgs sector, and the mixing effects on the SM-like Higgs boson. In section 3, we consider the case with a light singlet scalar, for which the SM-like Higgs boson can obtain the required mass via singlet-doublet mixing in TeV scale SUSY without large stop mixing. We will examine the range of mixing angles compatible with the current LHC and LEP data, and then discuss the implications of scalar mixing on the higgsino properties by using the fact that the Higgs boson masses and scalar mixing angles crucially depend on $\lambda$ and $\mu$. Section 4 is the conclusions.

\section{Higgs properties in the NMSSM}

In this section we briefly discuss how the Higgs sector is modified by the gauge singlet in the NMSSM. Then we summarize experimental and theoretical constraints on the Higgs sector, paying attention to how to arrange a $126 \mathrm{GeV}$ Higgs boson with SM-like properties in TeV scale SUSY.

\section{$2.1 \quad$ Higgs sector}

A singlet extension of the MSSM can always be described by the superpotential

$$
W=\lambda S H_{u} H_{d}+f(S)+(\text { MSSM Yukawa terms }),
$$

in the field basis where the gauge singlet $S$ has a minimal Kähler potential, $|S|^{2}$. Here the singlet superpotential $f$ is needed to avoid a phenomenologically unacceptable visible axion, and it has no dependence on the MSSM superfields at the renormalizable level. There are various NMSSM models classified by the form of $f$. In this paper we do not specify the form of $f$ as our results do not depend much on it, but we will assume no CP violation in the Higgs sector. 
After electroweak symmetry breaking (EWSB), the doublet Higgs bosons mix with the singlet boson via the couplings

$$
-\mathcal{L}_{\text {mix }}=\lambda^{2}|S|^{2}\left(\left|H_{u}\right|^{2}+\left|H_{d}\right|^{2}\right)+\left(A_{\lambda} \lambda S H_{u} H_{d}+\left(\partial_{S} f\right)^{*} \lambda H_{u} H_{d}+\text { h.c. }\right),
$$

where $A_{\lambda}$ is the soft SUSY breaking trilinear parameter. Using the EWSB conditions, one can find that the mass squared matrix for the neutral CP-even scalar bosons is written

$$
\left(\begin{array}{ccc}
m_{0}^{2}+\left(\lambda^{2} v^{2}-m_{Z}^{2}\right) \sin ^{2} 2 \beta & -\left(\lambda^{2} v^{2}-m_{Z}^{2}\right) \sin 2 \beta \cos 2 \beta & \lambda v(2 \mu-\Lambda \sin 2 \beta) \\
-\left(\lambda^{2} v^{2}-m_{Z}^{2}\right) \sin 2 \beta \cos 2 \beta & -\left(\lambda^{2} v^{2}-m_{Z}^{2}\right) \sin ^{2} 2 \beta+\frac{2 b}{\sin 2 \beta} & \lambda v \Lambda \cos 2 \beta \\
\lambda v(2 \mu-\Lambda \sin 2 \beta) & \lambda v \Lambda \cos 2 \beta & m_{\hat{s}}^{2}
\end{array}\right)
$$

in the basis $(\hat{h}, \hat{H}, \hat{s})$ defined by

$$
\begin{aligned}
\hat{h} & =\sqrt{2}\left(\left(\operatorname{Re} H_{d}^{0}-v \cos \beta\right) \cos \beta+\left(\operatorname{Re} H_{u}^{0}-v \sin \beta\right) \sin \beta\right), \\
\hat{H} & =\sqrt{2}\left(\left(\operatorname{Re} H_{d}^{0}-v \cos \beta\right) \sin \beta-\left(\operatorname{Re} H_{u}^{0}-v \sin \beta\right) \cos \beta\right), \\
\hat{s} & =\sqrt{2}(\operatorname{Re} S-\langle|S|\rangle),
\end{aligned}
$$

with $\left\langle\left|H_{u}^{0}\right|\right\rangle=v \sin \beta$ and $\left\langle\left|H_{d}^{0}\right|\right\rangle=v \cos \beta$ for $v \simeq 174 \mathrm{GeV}$. Here the effective higgsino mass parameter $\mu$, the Higgs scalar $b$-term, and the mixing parameter $\Lambda$ are determined by

$$
\begin{aligned}
\mu & =\lambda\langle S\rangle, \\
b & =A_{\lambda} \lambda\langle S\rangle+\lambda\left\langle\partial_{S} f\right\rangle^{*}, \\
\Lambda & =A_{\lambda}-\left\langle\partial_{S}^{2} f\right\rangle^{*} .
\end{aligned}
$$

If there is no mixing, $\hat{h}$ acts exactly like the SM Higgs boson with a mass determined by $m_{0}$ and $\lambda$. Including radiative corrections, which mainly come from top and stop loops [33-35], $m_{0}$ reads

$$
m_{0}^{2}=m_{Z}^{2}+\frac{3 m_{t}^{4}}{4 \pi^{2} v^{2}} \ln \left(\frac{m_{\tilde{t}}^{2}}{m_{t}^{2}}\right)+\frac{3 m_{t}^{4}}{4 \pi^{2} v^{2}}\left(X_{t}^{2}-\frac{1}{12} X_{t}^{4}\right)+\cdots
$$

where $m_{\tilde{t}}$ is the stop mass, and $X_{t}=\left(A_{t}-\mu \cot \beta\right) / m_{\tilde{t}}$ is the stop mixing parameter. Note that $m_{0}$ determines how heavy the SM-like Higgs boson can be within the MSSM, as it basically corresponds to the mass at large $\tan \beta$ in the decoupling limit of MSSM. ${ }^{1}$ There can be sizable radiative corrections also to other elements in the mass matrix, which lead to shifts of $b, \Lambda$, and $m_{\hat{s}}$. The mass eigenstates are found by diagonalizing the mass matrix (2.3), which introduces three mixing angles, $\theta_{i}$ :

$$
\left(\begin{array}{c}
h \\
H \\
s
\end{array}\right) \equiv\left(O_{\alpha \hat{\beta}}\right)\left(\begin{array}{c}
\hat{h} \\
\hat{H} \\
\hat{s}
\end{array}\right)=\left(\begin{array}{ccc}
c_{1} c_{2} & -s_{1} & -c_{1} s_{2} \\
c_{2} c_{3} s_{1}-s_{2} s_{3} & c_{1} c_{3} & -c_{3} s_{1} s_{2}-c_{2} s_{3} \\
c_{3} s_{2}+c_{2} s_{1} s_{3} & c_{1} s_{3} & c_{2} c_{3}-s_{1} s_{2} s_{3}
\end{array}\right)\left(\begin{array}{c}
\hat{h} \\
\hat{H} \\
\hat{s}
\end{array}\right),
$$

\footnotetext{
${ }^{1}$ There is an additional contribution from Higgs-singlino-higgsino loops, which is insensitive to $\tan \beta$, and can increase $m_{0}$ by a few $\mathrm{GeV}$ if both the singlino and higgsino are around the weak scale [36, 37].
} 
for $c_{i}=\cos \theta_{i}$ and $s_{i}=\sin \theta_{i}$, where $\alpha=\{h, H, s\}$ and $i=\{1,2,3\}$. The Lagrangian parameters are written in terms of mass eigenvalues and mixing angles $[13,14]$. Particularly important are the relation for $m_{0}$,

$$
m_{0}^{2}=m_{h}^{2}+\left(m_{H}^{2}-m_{h}^{2}\right) O_{H \hat{h}}\left(O_{H \hat{h}}+O_{H \hat{H}} \tan 2 \beta\right)-\left(m_{h}^{2}-m_{s}^{2}\right) O_{s \hat{h}}\left(O_{s \hat{h}}+O_{s \hat{H}} \tan 2 \beta\right),
$$

and the relations for $\lambda$ and $\mu$,

$$
\begin{aligned}
\lambda^{2} v^{2} & =m_{Z}^{2}-\frac{2}{\sin 4 \beta}\left(\left(m_{H}^{2}-m_{h}^{2}\right) O_{H \hat{h}} O_{H \hat{H}}-\left(m_{h}^{2}-m_{s}^{2}\right) O_{s \hat{h}} O_{s \hat{H}}\right), \\
\lambda v \mu & =\frac{1}{2}\left(m_{H}^{2}-m_{h}^{2}\right) O_{H \hat{s}}\left(O_{H \hat{h}}+O_{H \hat{H}} \tan 2 \beta\right)-\frac{1}{2}\left(m_{h}^{2}-m_{s}^{2}\right) O_{s \hat{s}}\left(O_{s \hat{h}}+O_{s \hat{H}} \tan 2 \beta\right) .
\end{aligned}
$$

These relations allow us to translate the constraints on $m_{0}, \lambda$, and $\mu$ into the constraints on the mass eigenvalues $m_{\alpha}$ and the mixing angles $\theta_{i}$, and vice versa.

\subsection{SM-like Higgs boson}

We identify $h$ as the scalar particle discovered at the LHC since it has properties close to those of the SM Higgs boson for small mixing. It interacts with SM particles via

$$
\begin{aligned}
\mathcal{L}_{\mathrm{eff}}= & C_{V} \frac{\sqrt{2} m_{W}^{2}}{v} h W_{\mu}^{+} W_{\mu}^{-}+C_{V} \frac{m_{Z}^{2}}{\sqrt{2} v} h Z_{\mu} Z_{\mu}-C_{f} \frac{m_{f}}{\sqrt{2} v} h \bar{f} f \\
& +C_{g} \frac{\alpha_{s}}{12 \sqrt{2} \pi v} h G_{\mu \nu}^{a} G_{\mu \nu}^{a}+C_{\gamma} \frac{\alpha}{\sqrt{2} \pi v} h A_{\mu \nu} A_{\mu \nu}
\end{aligned}
$$

around the weak scale. Here the Higgs couplings to the vector bosons and the SM fermions $f$ read

$$
C_{V}=c_{1} c_{2}, \quad C_{t}=c_{1} c_{2}+s_{1} \cot \beta, \quad C_{b}=C_{\tau}=c_{1} c_{2}-s_{1} \tan \beta,
$$

at tree-level, and so $h$ has $C_{V}=C_{f}=1$ in the limit of vanishing mixing angles. On the other hand, the couplings to massless gluons and photons are radiatively induced mainly from the $W$-boson and top-quark loops,

$$
\begin{aligned}
& C_{g} \simeq 1.03 C_{t}-0.06 C_{b}+\delta C_{g}, \\
& C_{\gamma} \simeq 0.23 C_{t}-1.04 C_{V}+\delta C_{\gamma},
\end{aligned}
$$

where $\delta C_{g}$ and $\delta C_{\gamma}$ are the contributions from superparticle loops. The SUSY contribution $\delta C_{g}$ can be sizable if the stops are relatively light, and is approximately estimated as

$$
\delta C_{g} \approx \frac{1}{4}\left(2-X_{t}^{2}\right) \frac{m_{t}^{2}}{m_{\tilde{t}}^{2}} C_{t}+\cdots,
$$

for small mass splitting between the two stops [38, 39]. In the presence of scalar mixing, the Higgs coupling to photons receives a contribution from the chargino loops [40],

$$
\delta C_{\gamma} \approx-0.17 \frac{\lambda v}{|\mu|} \cos \theta_{1} \sin \theta_{2}+\frac{1}{18}\left(2-X_{t}^{2}\right) \frac{m_{t}^{2}}{m_{\tilde{t}}^{2}} C_{t}+\cdots,
$$


assuming small mixing between the charged wino and higgsinos for simplicity. Here the first term comes from the charged-higgsino loops, and it either enhances or reduces the Higgs coupling to photons depending on the singlet-doublet mixing $\theta_{2}$. The second term is the contribution from the stop loops, and the ellipsis includes other SUSY contributions, which are small unless one considers large left-right mixing of the third generation sfermions, or a small mass around the weak scale for the charged Higgs boson.

The Higgs sector is constrained by the Higgs boson data from the LHC experiments. The signal rate of $h$ at the LHC can be estimated in terms of the effective Higgs couplings by using the well-known production and decay properties of the SM Higgs boson [41]. The signal strength normalized by the SM value is given by

$$
\begin{aligned}
R_{V V}^{\text {incl }} & =\frac{\sigma(p p \rightarrow h)}{\left.\sigma(p p \rightarrow h)\right|_{\mathrm{SM}}} \times \frac{\operatorname{Br}(h \rightarrow V V)}{\left.\operatorname{Br}(h \rightarrow V V)\right|_{\mathrm{SM}}} \\
& \simeq \frac{\left(0.92 C_{g}^{2}+0.12 C_{V}^{2}\right) C_{V}^{2}}{0.62 C_{b}^{2}+0.26 C_{V}^{2}+0.12 C_{t}^{2}},
\end{aligned}
$$

for the inclusive $W W / Z Z$ channel, where we have assumed that the Higgs decay rate into non-SM particles is negligible. For other channels, one finds

$$
\begin{aligned}
& R_{b b}^{\mathrm{incl}}=R_{\tau \tau}^{\mathrm{incl}}=\frac{C_{b}^{2}}{C_{V}^{2}} R_{V V}^{\mathrm{incl}}, \\
& R_{\gamma \gamma}^{\mathrm{incl}} \simeq \frac{1.49 C_{\gamma}^{2}}{C_{V}^{2}} R_{V V}^{\mathrm{incl}} .
\end{aligned}
$$

As it should be, the NMSSM leads to $R_{x x}^{\text {incl }}=1$ for each channel in the limit that the mixing angles vanish and the superparticles are decoupled with heavy masses, i.e. for $\theta_{i}=0$ and $\delta C_{g}=\delta C_{\gamma}=0$.

Another important constraint comes from the observed Higgs boson mass, $m_{h} \simeq$ $126 \mathrm{GeV}$. In the MSSM, the upper limit of $m_{h}$ is set by $m_{0}$. Figure 1 shows the dependence of $m_{0}$ on the stop mass and mixing parameters, which is obtained using FeynHiggs 2.10 .0 [42-45]. To get $m_{h} \simeq 126 \mathrm{GeV}$ within the MSSM, one needs heavy stops above about $7 \mathrm{TeV}$, or large stop mixing around $X_{t}^{2}=6$. On the other hand, the NMSSM can accommodate a $126 \mathrm{GeV}$ Higgs boson with SM-like properties for stops around $1 \mathrm{TeV}$ or even below, without relying on large stop mixing. This is because $m_{h}$ can be enhanced by the additional effects associated with the gauge singlet $S$. One effect is the tree-level $F$ term contribution, $\Delta m^{2}=\lambda^{2} v^{2} \sin ^{2} 2 \beta$, which becomes sizable at large $\lambda$ and small $\tan \beta$. Another effect arises from the mixing with the singlet scalar.

In this paper we focus on the supersymmetric SM with stops around $1 \mathrm{TeV}$ or below, as would be expected if SUSY is to stabilize the weak scale against large radiative corrections. The region of our interest is around and inside the red dashed box in figure 1. Especially we will focus on the case with $m_{s}<m_{h}$ so that the mixing effect raises the Higgs boson mass $m_{h}$, and examine how much the Higgs sector is constrained by the current experimental data and theoretical considerations. 


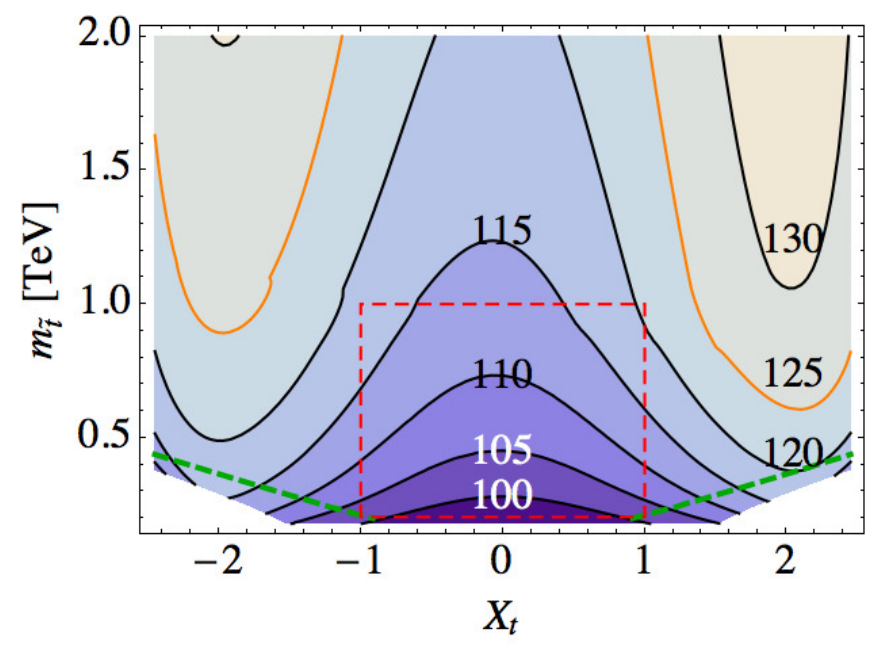

Figure 1. The dependence of $m_{0}$ on the stop mass and mixing parameter in the large $\tan \beta$ regime. In the MSSM $m_{0}$ sets the upper limit of the SM-like Higgs boson mass. We plot the constant contours of $m_{0}=105,110,115,120, \cdots, \mathrm{GeV}$, respectively, by taking $\mu=200 \mathrm{GeV}$ and $\tan \beta=40$. The lightest stop has a mass smaller than $200 \mathrm{GeV}$ in the region below the dashed green line. The region of our interest is inside and around the red dashed box.

\section{$3 \quad$ NMSSM with a light singlet scalar}

We first specify the range of $m_{0}$ and $m_{\alpha}$ in our scenario with a light singlet scalar, and then move on to the experimental constraints on the Higgs sector and their implications. The case of our interest is that the CP-even neutral Higgs sector has the mass spectrum

$$
\frac{1}{2} m_{h}<m_{s}<m_{h},
$$

with $m_{h} \simeq 126 \mathrm{GeV}$, while the superparticles including the stops are around or below TeV. Here we do not assume a particular mass spectrum for the superparticles or particular mediation mechanism of SUSY breaking. The lower bound on $m_{s}$ is to avoid the process $h \rightarrow s s$. For the heavier Higgs boson $H$, we consider

$$
350 \mathrm{GeV} \lesssim m_{H} \lesssim 1 \mathrm{TeV}
$$

taking into account the experimental constraint from $b \rightarrow s \gamma$, which requires the charged Higgs scalar to be heavier than about $350 \mathrm{GeV}$ barring cancellation with other superparticle contributions [46].

The NMSSM can explain the observed Higgs boson mass even for $m_{0}$ around or below $120 \mathrm{GeV}$, i.e. for stops having $m_{\tilde{t}} \lesssim 1 \mathrm{TeV}$ and $X_{t}^{2} \lesssim 1$, because there are extra contributions associated with the singlet scalar. To be specific, we take

$$
100 \mathrm{GeV} \lesssim m_{0} \lesssim 120 \mathrm{GeV}
$$

keeping in mind that $m_{0}$ sets the upper limit of the SM-like Higgs boson mass in the MSSM, and has a dependence on the stop mass and mixing as plotted in figure 1. For the 
coupling $\lambda$, we impose

$$
0.01<\lambda<1
$$

at the weak scale. The upper bound is to ensure that the model remains perturbative up to the conventional GUT scale. This requires $\lambda$ to be smaller than $0.7-0.8$ at the weak scale, which is slightly relaxed in the presence of extra heavy particles charged under the SM gauge group [47, 48]. The perturbative bound can be relaxed further in $\mathrm{U}(1)$ gauge extensions [49] or in extensions with hidden gauge sector coupled to $S$ [37]. On the other hand, the LEP constraint on the chargino mass requires $\mu$ larger than about $100 \mathrm{GeV}$ [32], implying that the singlet scalar has a VEV above $\lambda^{-1} \times 100 \mathrm{GeV}$. We have put a mild lower bound on $\lambda$ following that the singlet scalar would not have a VEV much larger than $\mathrm{TeV}$ in low scale SUSY.

Finally it should be noted that the relation (2.8) and (2.9) lead to

$$
\lambda^{2} v^{2}=m_{Z}^{2}+\frac{1}{\sin ^{2} 2 \beta}\left(\left(m_{h}^{2}-m_{0}^{2}\right)-\left(m_{h}^{2}-m_{s}^{2}\right) O_{s \hat{h}}^{2}+\left(m_{H}^{2}-m_{h}^{2}\right) O_{H \hat{h}}^{2}\right) .
$$

Thus, for $m_{s}<m_{h}$, small $\lambda$ requires sizable $O_{s \hat{h}}^{2}$ and small $O_{H \hat{h}}^{2}$. This simply reflects the fact that $m_{h}$ receives a positive contribution both from the tree-level $F$-term potential associated with the singlet and the singlet-doublet mixing effect, whereas a negative contribution from the doublet-doublet mixing effect.

\subsection{LHC constraints on Higgs mixing}

The Higgs couplings to SM fermions and vector bosons (2.12) are fixed by $\theta_{1}, \theta_{2}$, and $\tan \beta$, while the couplings to gluons and photons (2.13) can receive an additional sizable contribution from superparticle loops. This implies that the signal rate of the SM-like Higgs boson is a function written

$$
R_{x x}=R_{x x}\left(\theta_{1}, \theta_{2}, \tan \beta, \delta C_{g}, \delta C_{\gamma}\right),
$$

for each decay channel, $h \rightarrow x x$, with $x=\{W, Z, b, \tau, \gamma\}$. Hence the Higgs signal strength measured at the LHC puts a constraint on the mixing angle $\theta_{1}$ and $\theta_{2}$. To examine the constraint, one also needs to estimate $\delta C_{g}$ and $\delta C_{\gamma}$. The stop searches at the LHC suggest that stop mass lighter than about $600 \mathrm{GeV}$ is excluded depending on the mass of the lightest neutralino if the stop is kinematically allowed to decay into the top quark and the lightest neutralino [50-52]. Taking this into account, we separate two cases according to the stop mass, and find $m_{0}$ and $\delta C_{g}$ to be

$$
\begin{aligned}
& 600 \mathrm{GeV} \lesssim m_{\tilde{t}} \lesssim 1 \mathrm{TeV} \quad: 105 \mathrm{GeV} \lesssim m_{0} \lesssim 120 \mathrm{GeV}, \delta C_{g} \lesssim 0.04 C_{t}, \\
& 200 \mathrm{GeV} \lesssim m_{\tilde{t}} \lesssim 600 \mathrm{GeV}: 100 \mathrm{GeV} \lesssim m_{0} \lesssim 115 \mathrm{GeV}, \delta C_{g} \lesssim 0.21 C_{t},
\end{aligned}
$$

taking $X_{t}^{2} \lesssim 1$. On the other hand, the Higgs coupling to photons receives SUSY contributions,

$$
\left|\delta C_{\gamma}\right| \lesssim 0.3\left|\cos \theta_{1} \sin \theta_{2}\right|+\frac{2}{9} \delta C_{g}
$$




\begin{tabular}{|c|c|c|c|c|c|c|}
\hline & $R_{W W}^{\text {incl }}$ & $R_{Z Z}^{\text {incl }}$ & $R_{b b}^{\mathrm{VH} / \mathrm{VBF}}$ & $R_{\tau \tau}^{V H}$ & $R_{\gamma \gamma}^{X}$ & $R_{\gamma \gamma}^{Y}$ \\
\hline$\overline{\text { ATLA }}$ & $0.99_{-0.28}^{+0.31}$ & $1.43_{-0.35}^{+0.40}$ & \multicolumn{2}{|c|}{$1.09_{-0.32}^{+0.36 *}$} & $1.49 \pm 0.36$ & $0.61 \pm 0.75$ \\
\hline CMS & $68 \pm 0.20$ & $92 \pm 0.28$ & $1.15 \pm 0.62$ & $1.10 \pm 0$ & $1.42 \pm 0.31$ & $0.89 \pm 0.61$ \\
\hline
\end{tabular}

Table 1. The summary of the Higgs signal rates. These are evaluated at $m_{h}=125.5 \mathrm{GeV}$ for the ATLAS while $m_{h}=125.7 \mathrm{GeV}$ for the CMS. The number with asterisk is taken from ref. [58], and the others from refs. [53, 55-57].

from the chargino and stop loops, where we have used the relation (2.15) taking $\lambda<1$ as limited by the perturbativity constraint, and $|\mu|>100 \mathrm{GeV}$ considering the LEP bound on the chargino mass.

Let us examine the range of Higgs mixing compatible with the current experimental data reported by the ATLAS [53-55] and CMS $[56,57]$ collaborations, respectively. For the decay modes $h \rightarrow W W^{*}$ and $Z Z^{*}$, we consider the signal rate in the inclusive channel assuming that it is dominated by the gluon-gluon fusion $(\mathrm{ggF})$ production. For the fermionic modes, we focus on the vector boson fusion (VBF) and vector boson associated $(\mathrm{VH})$ production. On the other hand, the signal rate in the diphoton mode requires a more careful treatment because the reported data suggests a correlation between the diphoton rates in the ggF and VBF cannel. To employ a $\chi^{2}$ analysis, we define the measures

$$
\begin{aligned}
& R_{\gamma \gamma}^{X}=1+\left(R_{\gamma \gamma}^{\mathrm{ggF} / \mathrm{ttH}}-1\right) \cos \varphi+\left(R_{\gamma \gamma}^{\mathrm{VH} / \mathrm{VBF}}-1\right) \sin \varphi, \\
& R_{\gamma \gamma}^{Y}=1-\left(R_{\gamma \gamma}^{\mathrm{ggF} / \mathrm{ttH}}-1\right) \sin \varphi+\left(R_{\gamma \gamma}^{\mathrm{VH} / \mathrm{VBF}}-1\right) \cos \varphi,
\end{aligned}
$$

and take $\cos \varphi=0.98$ for the ATLAS data and 0.97 for the CMS data so that $R_{\gamma \gamma}^{X}$ and $R_{\gamma \gamma}^{Y}$ can be treated as independent. Table 1 summarizes the Higgs signal rates we will use in the analysis. Note that the signal rate normalized by the SM prediction is given by

$$
\begin{aligned}
R_{b b}^{\mathrm{VH} / \mathrm{VBF}} & \simeq \frac{C_{V}^{2} C_{b}^{2}}{0.62 C_{b}^{2}+0.26 C_{V}^{2}+0.12 C_{t}^{2}}, \\
R_{\gamma \gamma}^{\mathrm{VH} / \mathrm{VBF}} & \simeq \frac{1.52 C_{V}^{2} C_{\gamma}^{2}}{0.62 C_{b}^{2}+0.26 C_{V}^{2}+0.12 C_{t}^{2}},
\end{aligned}
$$

for $m_{h} \simeq 126 \mathrm{GeV}$. In the analysis we include the SUSY contributions $\delta C_{g}$ and $\delta C_{\gamma}$ lying in the range indicated above, and minimize $\chi^{2}$ at each point on the $\left(\theta_{1}, \theta_{2}\right)$ plane assuming a Gaussian distribution.

Figure 2 illustrates which region of $\left(\theta_{1}, \theta_{2}\right)$ is compatible with the current LHC data on the Higgs boson. The $\hat{h}$ fraction in $h$ is larger than 0.5 in the region between the two dotdashed blue curves, making $h$ SM-like. The dark and light orange regions are preferred at the $68 \%$ and $95 \%$ CL, respectively, by the ATLAS (upper) and CMS (lower) measurements. For comparison, we also plot the $68 \%$ (outer brown circle) and 95\% CL (inner brown circle) preferred region for the case with vanishing $\delta C_{g}$ and $\delta C_{\gamma}$. One can see that sizable scalar mixing is compatible with the current LHC data, and superparticle contributions to $C_{g}$ and $C_{\gamma}$ slightly enlarge the allowed region. In addition there are a couple of things to note. The shaded region is not symmetric under $\theta_{2} \rightarrow-\theta_{2}$ because the Higgs coupling to photons receives a contribution from the chargino loops combined with the singlet-doublet 

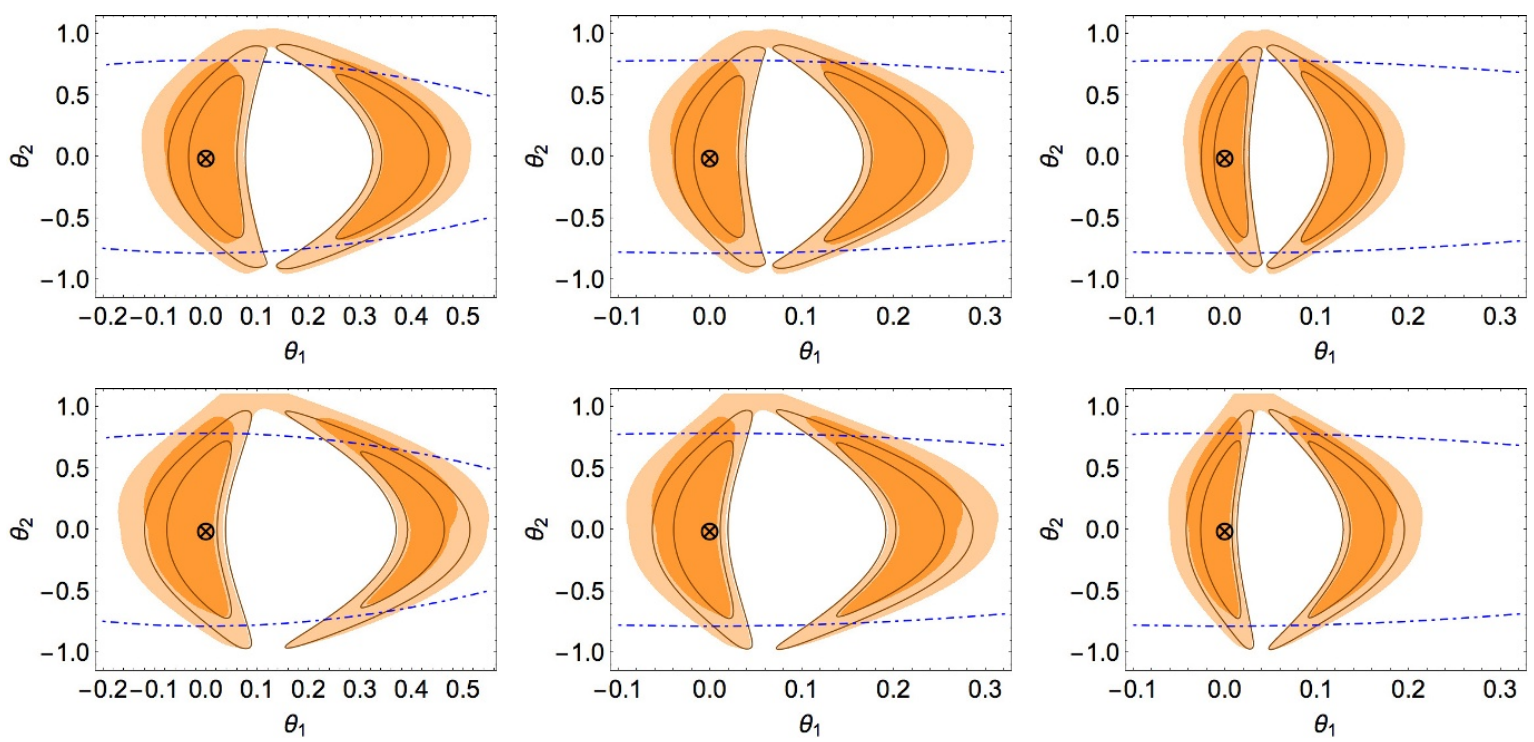

Figure 2. Higgs mixing compatible with the LHC data. We show the region of $\left(\theta_{1}, \theta_{2}\right)$ preferred at the $68 \%$ (dark orange) and $95 \% \mathrm{CL}$ (light orange) by the ATLAS (upper) and the CMS (lower) results, respectively. Here we have taken $m_{h}=126 \mathrm{GeV}$, and $\tan \beta=5,10,15$ for the left, middle, right panel. The $\hat{h}$ fraction in $h$, which is given by $O_{h \hat{h}}^{2}$ and determines how close $h$ is to the SM Higgs boson, is larger than 0.5 in the region between two dot-dashed blue curves. For comparison, we also plot the $68 \%$ (outer brown circle) and 95\% CL (inner brown circle) preferred region in the limit that the superparticles are very heavy, i.e. the case with $\delta C_{g}=\delta C_{\gamma}=0$.

mixing. For given $\tan \beta$, there are two ranges of $\theta_{1}$ where $h$ can describe the observed data. One is around $\theta_{1}=0$, and the other is around $\theta_{1}=\arctan (2 / \tan \beta)$ [13, 14]. This is understood from the fact that the Higgs decay $h \rightarrow b \bar{b}$ occurs through the effective coupling $C_{b}=c_{1} c_{2}-s_{1} \tan \beta$, and it should be the main decay mode in order to explain the LHC results [59]. Hence one needs either $C_{b} \sim 1$ or $C_{b} \sim-1$. The former is the case in the region around the origin, $\left(\theta_{1}, \theta_{2}\right)=(0,0)$. In the latter case, which is obtained in the region with $\tan \theta_{1}=2 / \tan \beta$ and small $\theta_{2}$, the sign of the Higgs coupling to down-type fermions is opposite to that of the SM Higgs boson, and consequently the bottom and top quark loops give the same sign of contributions to the Higgs coupling to gluons. ${ }^{2}$ Finally we note that the sensitivity of $C_{b}$ to $\tan \beta$ results in that the preferred region gets smaller as $\tan \beta$ increase, as can be seen from the figure. The future run of the LHC and linear collider experiments will help us to clarify the viable region of $\left(\theta_{1}, \theta_{2}\right)$ more accurately, and could determine the sign of Higgs coupling to bottom-quark pairs.

\subsection{Implication of Higgs mixing on higgsino properties}

The Higgs sector is further constrained by the observed Higgs boson mass, and the LEP results on the Higgs search if the singlet-like Higgs boson is lighter than $114 \mathrm{GeV}$ [61]. Interestingly, combined with the constraints from the measured Higgs signal rate, these are found to put an upper bound on $\mu$, requiring higgsinos to be relatively light. The LEP

\footnotetext{
${ }^{2}$ See also ref. [60] for the discussion on a wrong-sign Yukawa coupling in two-Higgs-doublet model.
} 


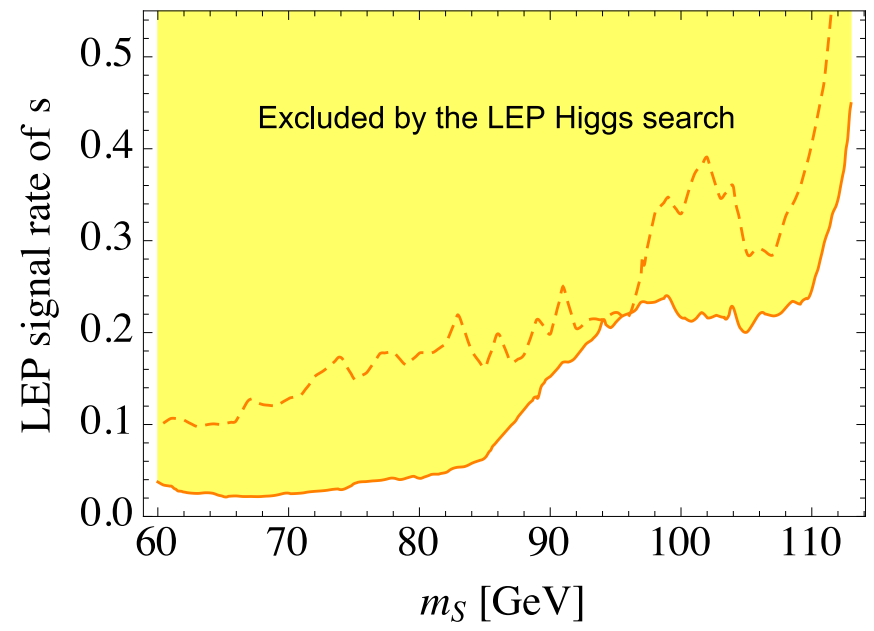

Figure 3. LEP constraints on the singlet-like Higgs boson $s$. The shaded region is excluded by the LEP result on Higgs searches in the channel, $e^{+} e^{-} \rightarrow Z s \rightarrow Z b \bar{b}$. The dashed orange curve shows the LEP constraint on the production cross section of hadronically decaying Higgs bosons.

signal rate of $s$ relative to the SM prediction is given by

$$
R\left(e^{+} e^{-} \rightarrow Z s \rightarrow Z b \bar{b}\right)=O_{s \hat{h}}^{2} \times \operatorname{Br}(s \rightarrow b \bar{b}) .
$$

The singlet-like Higgs boson $s$ dominantly decays into bottom quarks for $2 m_{b}<m_{s}<$ $m_{h}$ in most of the region of mixing angles. Note however that $\operatorname{Br}(s \rightarrow b \bar{b})$ is highly suppressed when the $s b \bar{b}$ coupling vanishes, i.e. for the scalar mixing satisfying $s_{2} c_{3}+$ $s_{1} c_{2} s_{3}+c_{1} s_{3} \tan \beta=0$. Figure 3 shows the LEP constraints on the singlet-like Higgs boson. In the yellow-shaded region, the signal rate of $s$ in the channel $e^{+} e^{-} \rightarrow Z s \rightarrow Z b \bar{b}$ is above the LEP bound. The dashed orange curve is the LEP constraint on the production cross section of hadronically decaying Higgs bosons [62].

Let us use the relations (2.8), (2.9) and (2.10) to see why light higgsinos are favored in the NMSSM with a light singlet scalar when $h$ is arranged to have properties consistent with the observation. From (2.8) and (2.9), one obtains

$$
\begin{aligned}
& \left(1-\frac{\lambda^{2} v^{2}-m_{Z}^{2}}{m_{H}^{2}-m_{h}^{2}} \cos ^{2} 2 \beta+2 \frac{m_{h}^{2}-m_{s}^{2}}{m_{H}^{2}-m_{h}^{2}} O_{s \hat{h}} O_{s \hat{H}} \cot 2 \beta\right)\left(\lambda^{2} v^{2}-m_{Z}^{2}\right) \sin ^{2} 2 \beta \\
= & \left(m_{h}^{2}-m_{0}^{2}\right)-\left(1-\frac{m_{h}^{2}-m_{s}^{2}}{m_{H}^{2}-m_{h}^{2}} O_{s \hat{H}}^{2}\right)\left(m_{h}^{2}-m_{s}^{2}\right) O_{s \hat{h}}^{2} .
\end{aligned}
$$

Since we consider the case with $m_{H}^{2} \gg m_{h}^{2}$ and $\lambda<1$, the above is approximated as

$$
\left(\lambda^{2} v^{2}-m_{Z}^{2}\right) \sin ^{2} 2 \beta \simeq\left(m_{h}^{2}-m_{0}^{2}\right)-\left(m_{h}^{2}-m_{s}^{2}\right) O_{s \hat{h}}^{2},
$$

for $\tan \beta \ll\left(m_{H}^{2}-m_{h}^{2}\right) /\left(m_{h}^{2}-m_{s}^{2}\right)$. Hence the value of $\lambda$ is mainly fixed by $\tan \beta$ and the mixing angles through $O_{s \hat{h}}=c_{3} s_{2}+c_{2} s_{1} s_{3}$, but insensitively to the precise value of $m_{H}$, as long as the Higgs sector has $m_{H}^{2} \gg m_{h}^{2}$. 
The Higgs boson $h$ becomes more SM-like when the mixing parameters $O_{h \hat{H}}$ and $O_{h \hat{s}}$ get smaller. Expanded in powers of $O_{h \hat{H}}$ and $O_{h \hat{s}}$, the relation (2.10) is written

$$
\begin{aligned}
\lambda v \mu \simeq & \frac{m_{h}^{2}-m_{s}^{2}}{1+t_{3}^{2}}\left(\frac{\tan \beta}{\tan ^{2} \beta-1} t_{3}+\frac{1}{2} O_{h \hat{s}}+\frac{t_{3}}{2} O_{h \hat{H}}\right) \\
& +\frac{m_{H}^{2}-m_{h}^{2}}{1+t_{3}^{2}}\left(\frac{\tan \beta}{\tan ^{2} \beta-1}-\frac{t_{3}}{2} O_{h \hat{s}}+\frac{1}{2} O_{h \hat{H}}\right)\left(t_{3}+O_{h \hat{s}} O_{h \hat{H}}\right),
\end{aligned}
$$

at the leading order. Here $t_{3} \equiv \tan \theta_{3}$, and we have assumed that $t_{3}^{2}$ is not much larger than unity, for which $H$ has a sizable $\hat{H}$ component. In addition, the relation (2.9) allows us to estimate $O_{h \hat{H}}$ in terms of $O_{h \hat{s}}$ as

$$
O_{h \hat{H}} \simeq \epsilon+t_{3} O_{h \hat{s}}
$$

for $m_{H}^{2} \gg m_{h}^{2}$, neglecting terms in higher order in $O_{h \hat{s}}$. The parameter $\epsilon$ is given by

$$
\epsilon \approx-\frac{2}{\tan \beta} \frac{\lambda^{2} v^{2}-m_{Z}^{2}}{m_{H}^{2}-m_{h}^{2}}\left(1+t_{3}^{2}\right)
$$

and so it is much smaller than $1 / \tan \beta$. Plugging the above relation between mixing parameters into (3.14), one arrives at

$$
\mu \approx \frac{m_{h}^{2}-m_{s}^{2}}{2 \lambda v} O_{h \hat{s}}+\frac{m_{H}^{2}-m_{s}^{2}}{\lambda v} \frac{1}{\tan \beta} \frac{O_{h \hat{s}}\left(O_{h \hat{H}}-\epsilon\right)}{O_{h \hat{s}}^{2}+\left(O_{h \hat{H}}-\epsilon\right)^{2}},
$$

for nonzero $O_{h \hat{s}}$, as is required to increase $m_{h}$ via the singlet-doublet mixing. Finally the higgsino mass parameter is found to lie in the range

$$
|\mu| \lesssim \frac{m_{h}^{2}-m_{s}^{2}}{2 \lambda v}\left|O_{h \hat{s}}\right|+\frac{m_{H}^{2}-m_{s}^{2}}{\lambda v} \frac{1}{\tan \beta} \frac{\left|O_{h \hat{s}}\left(O_{h \hat{H}}-\epsilon\right)\right|}{O_{h \hat{s}}^{2}+\left(O_{h \hat{H}}-\epsilon\right)^{2}},
$$

with $\lambda$ approximately determined by the relation (3.13),

$$
\lambda^{2} \approx \frac{m_{Z}^{2}}{v^{2}}+\frac{\tan ^{2} \beta}{4}\left(\frac{m_{h}^{2}-m_{0}^{2}}{v^{2}}-\frac{m_{h}^{2}-m_{s}^{2}}{v^{2}} \frac{\left(O_{h \hat{s}}^{2}+\left(O_{h \hat{H}}-\epsilon\right) O_{h \hat{H}}\right)^{2}}{O_{h \hat{s}}^{2}+\left(O_{h \hat{H}}-\epsilon\right)^{2}}\right) .
$$

Therefore there is an upper limit on $\mu$, depending on how close $h$ is to the SM Higgs boson. Note that $\mu$ takes the maximum value when the mixing parameter $O_{h \hat{H}}$ has a value, $O_{h \hat{H}}^{2} \simeq O_{h \hat{s}}^{2}$, for sizable $O_{h \hat{s}}$ compatible with the LEP constraint. Here we have used that $\epsilon$ has a tiny value for $m_{H}^{2} \gg m_{h}^{2}$ and $\lambda<1$. Another important feature is that the upper bound on $\mu$ grows as $m_{s}$ decreases, because the right hand side of (3.18) increases while $\lambda$ decreases. However the LEP constraints on the singlet-doublet mixing become stringent when the singlet-like Higgs boson is light.

We are ready to analyze how strongly the higgsino mass parameter is constrained in the NMSSM with a light singlet scalar. Our strategy is to examine the value of $\mu$ on the $\left(\theta_{1}, \theta_{2}\right)$ plane for fixed $m_{0}, m_{\alpha}$, and $\tan \beta$. Then $\theta_{3}$ is determined by $(2.8)$, and subsequently one can compute $\mu$ and $\lambda$ using (2.9) and (2.10). Here we notice that there exist at most two values of $\theta_{3}$ satisfying the relation (2.8). If there are two solutions at a given point 

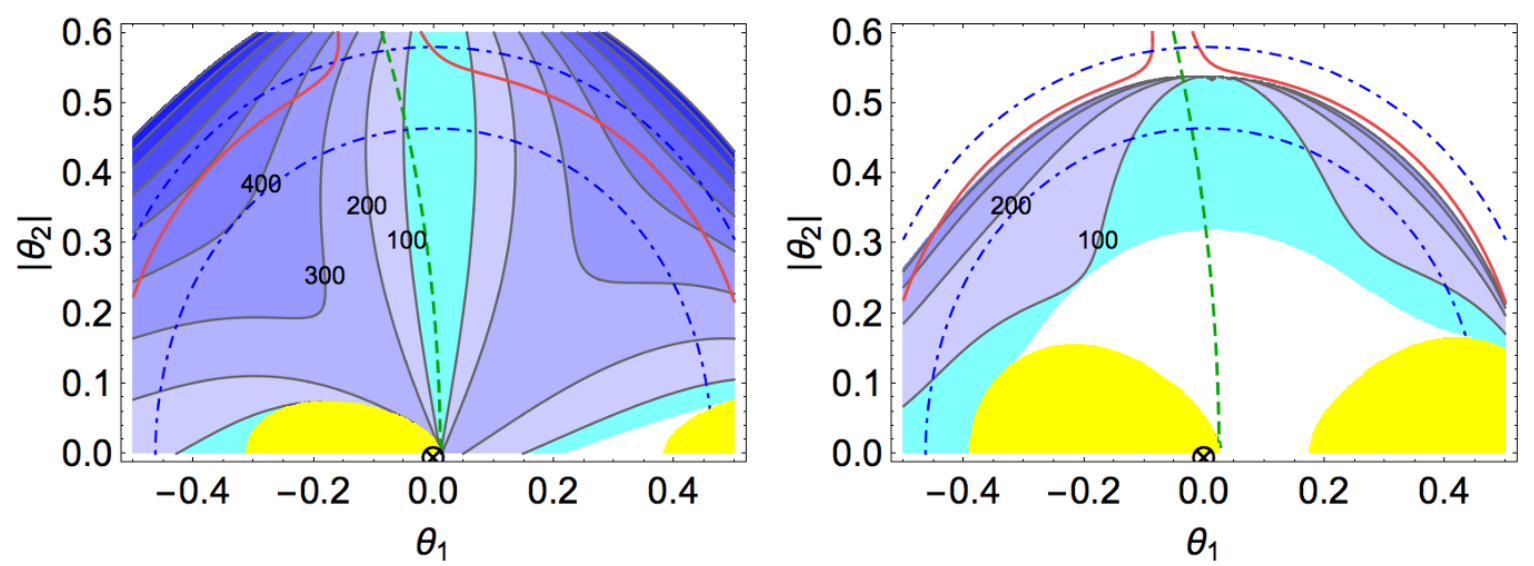

Figure 4. Higgsino mass parameter $\mu$ for $\tan \beta=5$ (left) and $\tan \beta=10$ (right) in the NMSSM with $m_{0}=120 \mathrm{GeV}, m_{h}=126 \mathrm{GeV}, m_{H}=600 \mathrm{GeV}$ and $m_{s}=95 \mathrm{GeV}$. On the $\left(\theta_{1}, \theta_{2}\right)$ plane, we plot the constant contours of $|\mu|=100,200,300,400, \cdots, \mathrm{GeV}$, respectively, with darker blue indicating larger $\mu$. The cyan-shaded region gives $\mu$ smaller than $100 \mathrm{GeV}$, and the unshaded region leads to $\lambda>1$. The yellow-shaded region is excluded because the relation (2.8) cannot be satisfied for a real value of $\theta_{3}$, while the region outside the red curve is excluded by the LEP constraints on the singlet-like Higgs boson $s$. The scalar mixing makes the $s b \bar{b}$ coupling vanish at tree level along the dashed green line, relaxing the LEP constraint on the signal rate of $s$. We also show the $\hat{h}$ fraction in $h: O_{h \hat{h}}^{2}=0.8(0.7)$ on the dot-dashed blue half-circle of smaller (larger) radius.

on the $\left(\theta_{1}, \theta_{2}\right)$ plane, we will take the value of $\theta_{3}$ that gives larger $\mu$. Figure 4 shows the higgsino mass parameter $|\mu|$ for $\tan \beta=5$ (left) and $\tan \beta=10$ (right) in the NMSSM with $m_{0}=120 \mathrm{GeV}, m_{h}=126 \mathrm{GeV}, m_{H}=600 \mathrm{GeV}$ and $m_{s}=95 \mathrm{GeV}$. For $m_{s}=95 \mathrm{GeV}$, the LEP constraint

$$
O_{s \hat{h}}^{2}=\left(s_{1} c_{2} c_{3}-s_{2} s_{3}\right)^{2} \lesssim \frac{0.24}{\operatorname{Br}(s \rightarrow b \bar{b})}
$$

is satisfied in the region inside the thick red curve. Note that along the dashed red line $s$ does not couple to the bottom quark at tree level, and thus the decay rate $s \rightarrow b \bar{b}$ is highly suppressed. As discussed above, large $\mu$ favors small $m_{s}$, but the LEP constraint becomes stronger as $s$ gets lighter. The contours of $|\mu|=100,200,300,400, \cdots, \mathrm{GeV}$ are shown by the solid gray lines, with darker blue indicating larger $\mu$. The cyan-shaded region gives $\mu$ smaller than $100 \mathrm{GeV}$ and so is in conflict with the LEP constraint on the chargino mass, while the yellow-shaded region is excluded since the relation (2.8) has no solution for real $\theta_{3}$. We also show the $\hat{h}$ fraction in the SM-like Higgs boson $h: O_{h \hat{h}}^{2}=0.8(0.7)$ on the dot-dashed blue half-circle of smaller (larger) radius. As can be seen from the figure, the higgsino mass is required to be small as $h$ becomes more SM-like, and $\tan \beta$ increases. In the unshaded region, $\lambda$ is above the perturbative bound, requiring new physics below the GUT scale. If one allows $\lambda>1$ at the weak scale, there appears additionally a viable region with $|\mu|>100 \mathrm{GeV}$, but only in the outer unshaded region where $h$ is less SM-like. Notice that the value of $\theta_{3}$ is fixed by solving the relation (2.8), and there are two solutions in the area except the yellow-shaded region. Inserting the two solutions into (2.9), one finds 

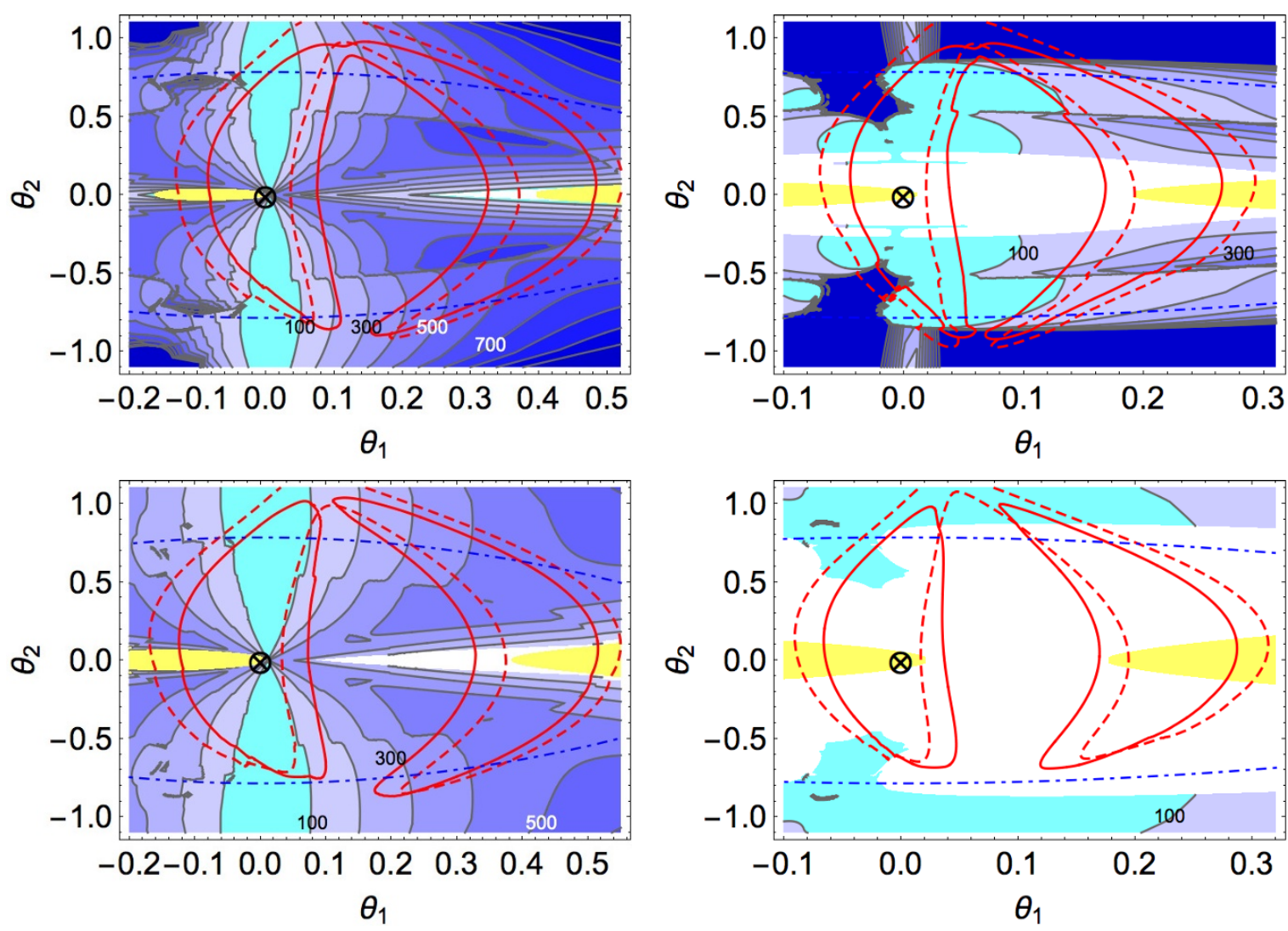

Figure 5. Upper bound on the higgsino mass parameter, $|\mu|_{\max }$, for $\tan \beta=5$ (left) and $\tan \beta=10$ (right) in the NMSSM with $m_{h}=126 \mathrm{GeV}, m_{H}=800 \mathrm{GeV}$, and $m_{s}$ taken to maximize $\mu$ under the LEP constraint. We have taken $m_{0}=120 \mathrm{GeV}(115 \mathrm{GeV})$ in the upper (lower) panel taking into account that $m_{0}$ increases and the stop contribution to the $g g h$ coupling decreases as the stop mass increases. We plot the constant contours of $|\mu|_{\max }=100,200,300,400, \cdots \mathrm{GeV}$ on the $\left(\theta_{1}, \theta_{2}\right)$ plane. The solid and dashed red circle are the region at preferred the $95 \% \mathrm{CL}$ by the ATLAS and CMS results, respectively. The $\hat{h}$ fraction in $h$ is larger than 0.5 in the region between two dot-dashed blue curves. The coupling $\lambda$ is above the perturbative bound in the unshaded region, while $|\mu|_{\max }<100 \mathrm{GeV}$ in the cyan-shaded region. The yellow-shaded region is excluded since there is no solution satisfying the relation (2.8).

that the value of $\lambda^{2}$ either keeps growing or changes sign when one crosses the boundary between the blue-shaded and outer unshaded region. Combined with the relation (2.10), this explains why $\mu$ is large near the boundary.

Let us continue to examine the maximum value of $\mu$ in the region of $\left(\theta_{1}, \theta_{2}\right)$ compatible with the current LHC data. This is done by varying $m_{s}$ for the given values of $m_{0}, m_{h}$, $m_{H}$ and $\tan \beta$. As was done above, the relation (2.8) is used to fix $\theta_{3}$ at each point, and then the relations (2.9) and (2.10) are combined to examine which value of $m_{s}$ maximizes $\mu$ under the LEP constraints on $s$ if $m_{s}<114 \mathrm{GeV}$. Before going into the analysis, we present an approximated expression for the upper bound on $\mu$ :

$$
|\mu|_{\max } \sim 350 \mathrm{GeV} \times\left(\frac{2\left|\theta_{1} \theta_{2}\right|}{\theta_{1}^{2}+\theta_{2}^{2}}\left(\frac{m_{H}}{800 \mathrm{GeV}}\right)^{2}\left(\frac{\tan \beta}{10}\right)^{-1}+0.1\left|\theta_{2}\right|\right),
$$


which is obtained from (3.18) for $\theta_{2} \neq 0$. Here $2\left|\theta_{1} \theta_{2}\right| /\left(\theta_{1}^{2}+\theta_{2}^{2}\right) \leq 1$, and it takes the maximum value at $\theta_{1}= \pm \theta_{2}$. The above expression is in the good agreement with the value evaluated from (2.8), (2.9) and (2.10) in the region of $\left(\theta_{1}, \theta_{2}\right)$ where $\lambda$ is below the perturbative bound and $h$ is SM-like, with $O_{h \hat{h}}^{2}>0.5$ and the signal rates compatible with the current LHC data. One can see that the upper bound on $\mu$ becomes stringent at large $\tan \beta$ and small $m_{H}$. We find that the higgsinos have masses around or below $300 \mathrm{GeV}$ in the $95 \%$ preferred region by the LHC data on the Higgs signal rates, as long as the heavy doublet Higgs boson has a mass, $m_{H} \lesssim 250 \sqrt{\tan \beta} \mathrm{GeV}$, and the singlet-like Higgs boson is consistent with the LEP constraints. Also important is the dependence of $|\mu|_{\max }$ on $m_{0}$. For fixed $m_{\alpha}$, the viable region with $\lambda<1$ is pushed far away from the origin on the $\left(\theta_{1}, \theta_{2}\right)$ plane if one takes small $m_{0}$ or large $\tan \beta$, because then the observed Higgs boson mass $m_{h} \simeq 126 \mathrm{GeV}$ requires large $\lambda$ or large singlet-doublet mixing. The Higgs coupling to photons receives a contribution from the charged-higgsino loops according to (2.15), which can be sizable for $\mu$ below a few hundred $\mathrm{GeV}$ in the region where the singlet-doublet mixing plays an important role in achieving $m_{h} \simeq 126 \mathrm{GeV}$.

Our results are summarized in figure 5 . The upper bound on the higgsino mass parameter, $|\mu|_{\max }$, is displayed on the $\left(\theta_{1}, \theta_{2}\right)$ plane for the NMSSM with $m_{h}=126 \mathrm{GeV}$ and $m_{H}=800 \mathrm{GeV}$, taking $\tan \beta=5$ and 10 in the left and right plot, respectively. Here we have taken $m_{0}=120 \mathrm{GeV}(115 \mathrm{GeV})$ in the upper (lower) panel taking into account two cases with stop mass above and below $600 \mathrm{GeV}$ as in (3.7). Note that $m_{0}$ increases with the stop mass. The constant contours of $|\mu|_{\max }=100,200,300,400, \cdots \mathrm{GeV}$ are shown in the gray lines. The solid and dashed red circles correspond to the $95 \%$ preferred regions by the ATLAS and CMS results, respectively. In the yellow-shaded region there is no real value of $\theta_{3}$ satisfying the relation (2.8). The value of $|\mu|_{\max }$ is less than $100 \mathrm{GeV}$ in the cyan-shaded region, while $\lambda$ is larger than unity in the unshaded region. In the region between two dot-dashed blue curves, the $\hat{h}$ fraction in $h$ is larger than 0.5 , and $|\mu|_{\max }$ decreases if one takes larger $\tan \beta$, because it is roughly proportional to $m_{H}^{2} / \tan \beta$. We also see that smaller $m_{0}$ or larger $\tan \beta$ pushes the region with $\lambda<1$ further away from the origin, thereby requiring smaller higgsino mass in the viable region where $h$ is SM-like and has properties compatible with the LHC results. As explained above, this is because $m_{h}$ is the sum of $m_{0}$ and the additional NMSSM contributions. Finally it is worth noting that, if one allows $\lambda>1$ at the weak scale, the blue and cyan area simply extend to the unshaded region. There is no change in our conclusion that the higgsinos have masses around or below $300 \mathrm{GeV}$ for $m_{H} \lesssim 250 \sqrt{\tan \beta} \mathrm{GeV}$ in the region compatible with the LHC and LEP constraints.

\subsection{CP-odd Higgs bosons}

Let us shortly discuss the CP-odd Higgs sector. The lightest CP-odd neutral Higgs boson $A$ interacts with SM particles through the doublet Higgs component, implying that there arise $A b b, Z h A$ and $Z s A$ coupling, but no $A W W$ and $A Z Z$ coupling at the tree-level $[5,6]$. 
The Higgs boson $A$ obtains a mass according to

$$
m_{A}^{2}=\frac{|b|}{\sin 2 \beta}+\frac{1}{2} m_{\hat{s}}^{\prime 2}-\sqrt{\left(\frac{|b|}{\sin 2 \beta}-\frac{1}{2} m_{\hat{s}}^{\prime 2}\right)^{2}+\lambda^{2} v^{2} A_{\lambda}^{2}},
$$

where $m_{\hat{s}}^{\prime}$ has a value different from $m_{\hat{s}}$ appearing in the CP-even Higgs mass matrix (2.3) because the singlet scalar receives explicit $\mathrm{U}(1)_{S}$ breaking mass contributions from the superpotential $f(S)$ and the associated soft SUSY breaking terms.

The case of our interest is that $A$ is singlet-like, and the doublet-like CP-odd Higgs boson is much heavier than $A$. Then the $h A A$ coupling is approximately given by $\lambda^{2} v$, and the $A b b$ couplings is estimated as

$$
y_{A b b} \simeq y_{b} \tan \beta \sin \phi,
$$

with $y_{b}$ being the bottom quark Yukawa coupling. The mixing angle $\phi$ between CP-odd Higgs bosons is smaller than about $m_{s}^{2} / m_{H}^{2}$, and the $Z h A$ and $Z s A$ couplings vanish in the decoupling regime where one combination of $H_{u}$ and $H_{d}$ is much heavier than the weak scale. There are LEP constraints on the processes, $e^{+} e^{-} \rightarrow Z A \rightarrow Z b \bar{b}$, and $e^{+} e^{-} \rightarrow Z^{*} \rightarrow s A$ or $h A$, depending on the mass of $A$. Using the properties discussed above, one finds that these constraints can be avoided without difficulty when $A$ is singletlike. On the other hand, the Higgs signal rate at the LHC is modified by the process, $h \rightarrow A A^{*} \rightarrow 4 b$, if kinematically open. The branching fraction of this decay mode is however smaller than the decay via $h \rightarrow Z Z^{*} \rightarrow 4 b$ for $y_{A b b} \ll 1$ and $\lambda<1$.

\subsection{Neutralino sector}

The NMSSM neutralino sector includes the singlino, which modifies the property of the lightest neutralino crucially depending on the supersymmetric singlino mass. The singlet superpotential is written as

$$
f(S)=\xi S+m S^{2}+\kappa S^{3}
$$

neglecting terms suppressed by the cut-off scale of the theory. Here the tadpole and mass terms should be around or below $\mathrm{TeV}$ to achieve EWSB without severe fine-tuning. These terms are suppressed if one imposes a discrete symmetry such as $Z_{3}$, but with small explicit breaking so as to avoid the domain-wall problem [5, 6]. Another interesting and natural way is to incorporate the Peccei-Quinn symmetry solving the strong CP problem via the invisible axion, so that $S$ obtains small tadpole and mass terms only after the Peccei-Quinn symmetry is spontaneously broken [63-65].

The lightest neutralino $\chi$ interacts with the SM particles, and there are various experimental constraints on its couplings, in particular, on those to the SM-like Higgs boson and the $Z$-boson:

$$
\mathcal{L}_{\text {int }}=\frac{1}{2}\left(y_{\chi} h \bar{\psi} \psi+\text { h.c. }\right)+c_{\chi} \frac{m_{Z}}{v} \bar{\psi} \gamma^{\mu} \gamma^{5} \psi Z_{\mu}+\cdots,
$$


where $\psi^{T}=(\chi, \bar{\chi})$ is the four-component spinor, and the couplings are determined by the neutralino mixing parameters

$$
\begin{aligned}
y_{\chi}= & \left(g^{\prime} N_{1}\left(N_{4} \sin \beta-N_{3} \cos \beta\right)-g N_{2}\left(N_{4} \sin \beta-N_{3} \cos \beta\right)\right) \cos \theta_{1} \cos \theta_{2} \\
& -\sqrt{2} \lambda N_{5}\left(N_{4} \cos \beta+N_{3} \sin \beta\right) \cos \theta_{1} \cos \theta_{2}, \\
c_{\chi}= & N_{3}^{2}-N_{4}^{2},
\end{aligned}
$$

in the presence of scalar mixing. Here the lightest neutralino $\chi$ is composed by

$$
\chi=N_{1} \tilde{B}+N_{2} \tilde{W}^{3}+N_{3} \tilde{H}_{d}^{0}+N_{4} \tilde{H}_{u}^{0}+N_{5} \tilde{S},
$$

with $g$ and $g^{\prime}$ being the $\mathrm{SU}(2)$ and $\mathrm{U}(1)_{Y}$ gauge couplings, respectively. The mixing parameters are fixed by diagonalizing the mass matrix

$$
\left(\begin{array}{ccccc}
M_{\tilde{B}} & 0 & -\frac{g^{\prime} v}{\sqrt{2}} \cos \beta & \frac{g^{\prime} v}{\sqrt{2}} \sin \beta & 0 \\
0 & M_{\tilde{W}} & \frac{g v}{\sqrt{2}} \cos \beta & -\frac{g v}{\sqrt{2}} \sin \beta & 0 \\
-\frac{g^{\prime} v}{\sqrt{2}} \cos \beta & \frac{g v}{\sqrt{2}} \cos \beta & 0 & -\mu & -\lambda v \sin \beta \\
\frac{g^{\prime} v}{\sqrt{2}} \sin \beta & -\frac{g v}{\sqrt{2}} \sin \beta & -\mu & 0 & -\lambda v \cos \beta \\
0 & 0 & -\lambda v \sin \beta & -\lambda v \cos \beta & \left\langle\partial_{S}^{2} f\right\rangle
\end{array}\right),
$$

which is given in the basis, $\left(\tilde{B}, \tilde{W}, \tilde{H}_{d}^{0}, \tilde{H}_{u}^{0}, \tilde{S}\right)$, with $M_{\lambda}$ being the mass of the indicated gaugino.

Let us briefly discuss the constraints on the neutralino sector. Since the NMSSM with a light singlet scalar requires relatively light higgsinos, we pay our attention to the case where the lightest neutralino has a sizable higgsino component. The $h \chi \chi$ and $Z \chi \chi$ coupling are constrained by the LUX and XENON results on direct dark matter searches $[66,67]$

$$
\begin{aligned}
\sigma_{\mathrm{SI}} & \simeq 0.9 \times 10^{-44} \mathrm{~cm}^{2}\left(\frac{y_{\chi}}{0.1}\right)^{2}\left(\frac{m_{h}}{126 \mathrm{GeV}}\right)^{-4} \lesssim 0.8 \times 10^{-45} \mathrm{~cm}^{2}\left(\frac{\Omega_{\chi} h^{2}}{0.11}\right)^{-1}, \\
\sigma_{\mathrm{SD}} & \simeq 0.8 \times 10^{-40} \mathrm{~cm}^{2}\left(\frac{c_{\chi}}{0.1}\right)^{2} \lesssim 0.35 \times 10^{-39} \mathrm{~cm}^{2}\left(\frac{\Omega_{\chi} h^{2}}{0.11}\right)^{-1},
\end{aligned}
$$

for $\Omega_{\chi} h^{2}$ being the relic energy density of $\chi$. Here the upper limit on the spin-independent neutralino-nucleon cross section is for $m_{\chi}=33 \mathrm{GeV}$ [66], where it reaches the minimum, while the upper limit on the spin-dependent one is for $m_{\chi}=45 \mathrm{GeV}$ [68]. If $\chi$ constitutes the main component of dark matter, the above requires both the $h \chi \chi$ and $Z \chi \chi$ coupling to be smaller than about 0.1 unless $\chi$ is lighter than $10 \mathrm{GeV}$. For the NMSSM with relatively light higgsinos, such small couplings are obtained if $\chi$ is almost higgsino-like, or if the singlino or bino is much lighter than the higgsino. The experimental constraints from the direct dark matter search are relaxed if $\chi$ composes a portion of the dark matter, for which one may consider the gravitino, axino, and/or axion as the main component of dark matter. The $h \chi \chi$ coupling is further constrained by the LHC bound on the Higgs invisible decay if $2 m_{\chi}<m_{h}[69,70]$. In addition, the LEP experiment puts a constraint on the neutralino production if the sum of the lightest and the second lightest neutralino masses is below $209 \mathrm{GeV}$ [71]. 
We close this subsection by mentioning the relic abundance of the lightest neutralino in the NMSSM with R-parity conservation. If $\chi$ has a large higgsino component, the tchannel chargino-mediated process $\chi \chi \rightarrow W^{+} W^{-}$occurs with a large annihilation cross section for $m_{\chi}>m_{W}$ [72], and thus the dark matter of the Universe cannot be explained by the neutralino thermal relic alone. The process $\chi \chi \rightarrow h h$ or $s s$ can be also important if $m_{\chi}>m_{h}$. To get a sufficient relic density, one may consider sizable mixing with bino or singlino. Another way is to consider non-thermal production, or other dark matter candidates such as the gravitino, axino, and/or the axion. On the other hand, for the case where $\chi$ has a mass below $m_{W}$ but above $m_{h} / 2$, the thermal relic abundance of $\chi$ is too large if the s-channel $Z$-boson exchange dominates the neutralino annihilation. This can be avoided by Higgs resonant annihilation. One may instead rely on non-thermal production, or late-time entropy production diluting the neutralino abundance.

\section{Conclusions}

The SM-like Higgs boson discovered at the LHC places important constraints on the supersymmetric extensions of the SM. Extended to include a gauge singlet, the Higgs sector can naturally explain the observed Higgs boson mass within TeV scale SUSY. In this paper we have focused on the NMSSM scenario where the singlet scalar is below the weak scale so that singlet-doublet mixing enhances the mass of the SM-like Higgs boson, and examined the phenomenological consequences of scalar mixing. The current experimental data allows sizable scalar mixing in the region around $\left(\theta_{1}, \theta_{2}\right)=(0,0)$ and $(2 / \tan \beta, 0)$. The two regions are distinguished by the sign of the Higgs coupling to down-type fermions.

The higgsino mass parameter and the singlet coupling to Higgs bilinear have a crucial dependence on the Higgs boson masses and mixing angles. Using the relations among them we found that the scalar mixing compatible with the LHC results on the SM-like Higgs boson leads to relatively light higgsinos, around or below a few hundred $\mathrm{GeV}$, as long as the heavy doublet Higgs boson has a mass smaller than about $250 \sqrt{\tan \beta} \mathrm{GeV}$, and the singlet-like Higgs boson is consistent with the LEP constraints. Also important is that the charged-higgsino loops combined with singlet-doublet mixing give a contribution to the Higgs coupling to photon, which has either sign and can be sizable when the higgsinos are light. The future run of the LHC and future linear collider experiments will clarify the viable range of mixing with higher accuracy, and could detect the singlet-like Higgs boson while probing the structure of the Higgs sector.

\section{Acknowledgments}

KSJ is supported by IBS under the project code, IBS-R018-D1. YS is supported by Grantin-Aid for JSPS Fellows under the program number 26.3171, and Tohoku University Institute for International Advanced Research and Education. MY is supported by Grants-inAid for Scientific Research from the Ministry of Education, Science, Sports, and Culture (MEXT), Japan, No. 23104008 and No. 23540283. 
Open Access. This article is distributed under the terms of the Creative Commons Attribution License (CC-BY 4.0), which permits any use, distribution and reproduction in any medium, provided the original author(s) and source are credited.

\section{References}

[1] H.P. Nilles, Supersymmetry, Supergravity and Particle Physics, Phys. Rept. 110 (1984) 1 [INSPIRE].

[2] H.E. Haber and G.L. Kane, The Search for Supersymmetry: Probing Physics Beyond the Standard Model, Phys. Rept. 117 (1985) 75 [INSPIRE].

[3] ATLAS collaboration, Observation of a new particle in the search for the Standard Model Higgs boson with the ATLAS detector at the LHC, Phys. Lett. B 716 (2012) 1 [arXiv: 1207.7214] [INSPIRE].

[4] CMS collaboration, Observation of a new boson at a mass of $125 \mathrm{GeV}$ with the CMS experiment at the LHC, Phys. Lett. B 716 (2012) 30 [arXiv:1207.7235] [INSPIRE].

[5] M. Maniatis, The Next-to-Minimal Supersymmetric extension of the Standard Model reviewed, Int. J. Mod. Phys. A 25 (2010) 3505 [arXiv:0906.0777] [InSPIRE].

[6] U. Ellwanger, C. Hugonie and A.M. Teixeira, The Next-to-Minimal Supersymmetric Standard Model, Phys. Rept. 496 (2010) 1 [arXiv:0910.1785] [INSPIRE].

[7] L.J. Hall, D. Pinner and J.T. Ruderman, A Natural SUSY Higgs Near 126 GeV, JHEP 04 (2012) 131 [arXiv:1112.2703] [INSPIRE].

[8] Z. Kang, J. Li and T. Li, On Naturalness of the MSSM and NMSSM, JHEP 11 (2012) 024 [arXiv: 1201.5305] [INSPIRE].

[9] G.G. Ross, K. Schmidt-Hoberg and F. Staub, The Generalised NMSSM at One Loop: Fine Tuning and Phenomenology, JHEP 08 (2012) 074 [arXiv:1205.1509] [INSPIRE].

[10] S.F. King, M. Mühlleitner, R. Nevzorov and K. Walz, Natural NMSSM Higgs Bosons, Nucl. Phys. B 870 (2013) 323 [arXiv:1211.5074] [InSPIRE].

[11] R.T. D'Agnolo, E. Kuflik and M. Zanetti, Fitting the Higgs to Natural SUSY, JHEP 03 (2013) 043 [arXiv: 1212.1165] [InSPIRE].

[12] T. Gherghetta, B. von Harling, A.D. Medina and M.A. Schmidt, The Scale-Invariant NMSSM and the $126 \mathrm{GeV}$ Higgs Boson, JHEP 02 (2013) 032 [arXiv:1212.5243] [INSPIRE].

[13] K. Choi, S.H. Im, K.S. Jeong and M. Yamaguchi, Higgs mixing and diphoton rate enhancement in NMSSM models, JHEP 02 (2013) 090 [arXiv:1211.0875] [INSPIRE].

[14] K. Choi, S.H. Im, K.S. Jeong and M.-S. Seo, Higgs phenomenology in the Peccei-Quinn invariant NMSSM, JHEP 01 (2014) 072 [arXiv:1308.4447] [INSPIRE].

[15] R. Barbieri, L.J. Hall, A.Y. Papaioannou, D. Pappadopulo and V.S. Rychkov, An Alternative NMSSM phenomenology with manifest perturbative unification, JHEP 03 (2008) 005 [arXiv: 0712.2903] [INSPIRE].

[16] M. Badziak, M. Olechowski and S. Pokorski, New Regions in the NMSSM with a $125 \mathrm{GeV}$ Higgs, JHEP 06 (2013) 043 [arXiv:1304.5437] [INSPIRE]. 
[17] C. Beskidt, W. de Boer and D.I. Kazakov, A comparison of the Higgs sectors of the CMSSM and NMSSM for a 126 GeV Higgs boson, Phys. Lett. B 726 (2013) 758 [arXiv:1308.1333] [INSPIRE].

[18] U. Ellwanger and C. Hugonie, The semi-constrained NMSSM satisfying bounds from the LHC, LUX and Planck, JHEP 08 (2014) 046 [arXiv: 1405.6647] [INSPIRE].

[19] U. Ellwanger, Enhanced di-photon Higgs signal in the Next-to-Minimal Supersymmetric Standard Model, Phys. Lett. B 698 (2011) 293 [arXiv:1012.1201] [INSPIRE].

[20] U. Ellwanger, A Higgs boson near $125 \mathrm{GeV}$ with enhanced di-photon signal in the NMSSM, JHEP 03 (2012) 044 [arXiv: 1112.3548] [INSPIRE].

[21] J.-J. Cao, Z.-X. Heng, J.M. Yang, Y.-M. Zhang and J.-Y. Zhu, A SM-like Higgs near 125 GeV in low energy SUSY: a comparative study for MSSM and NMSSM, JHEP 03 (2012) 086 [arXiv:1202.5821] [INSPIRE].

[22] S. Munir, L. Roszkowski and S. Trojanowski, Simultaneous enhancement in $\gamma \gamma, b \bar{b}$ and $\tau^{+} \tau^{-}$ rates in the NMSSM with nearly degenerate scalar and pseudoscalar Higgs bosons, Phys. Rev. D 88 (2013) 055017 [arXiv: 1305.0591] [INSPIRE].

[23] G. Bélanger, U. Ellwanger, J.F. Gunion, Y. Jiang, S. Kraml et al., Higgs Bosons at 98 and $125 \mathrm{GeV}$ at LEP and the LHC, JHEP 01 (2013) 069 [arXiv:1210.1976] [INSPIRE].

[24] C. Cheung, S.D. McDermott and K.M. Zurek, Inspecting the Higgs for New Weakly Interacting Particles, JHEP 04 (2013) 074 [arXiv: 1302.0314] [INSPIRE].

[25] R. Barbieri, D. Buttazzo, K. Kannike, F. Sala and A. Tesi, Exploring the Higgs sector of a most natural NMSSM, Phys. Rev. D 87 (2013) 115018 [arXiv:1304.3670] [InSPIRE].

[26] R. Barbieri, D. Buttazzo, K. Kannike, F. Sala and A. Tesi, One or more Higgs bosons?, Phys. Rev. D 88 (2013) 055011 [arXiv: 1307.4937] [INSPIRE].

[27] G. Cacciapaglia, A. Deandrea, G.D. La Rochelle and J.-B. Flament, Searching for a lighter Higgs: parametrisation and sample tests, arXiv:1311.5132 [INSPIRE].

[28] Z. Kang, J. Li, T. Li, D. Liu and J. Shu, Probing the CP-even Higgs sector via $\mathrm{H}_{3} \rightarrow \mathrm{H}_{2} \mathrm{H}_{1}$ in the natural next-to-minimal supersymmetric standard model, Phys. Rev. D 88 (2013) 015006 [arXiv: 1301.0453] [INSPIRE].

[29] D.G. Cerdeno, P. Ghosh and C.B. Park, Probing the two light Higgs scenario in the NMSSM with a low-mass pseudoscalar, JHEP 06 (2013) 031 [arXiv:1301.1325] [INSPIRE].

[30] N.D. Christensen, T. Han, Z. Liu and S. Su, Low-Mass Higgs Bosons in the NMSSM and Their LHC Implications, JHEP 08 (2013) 019 [arXiv:1303.2113] [INSPIRE].

[31] U. Ellwanger, Higgs pair production in the NMSSM at the LHC, JHEP 08 (2013) 077 [arXiv: 1306.5541] [INSPIRE].

[32] Particle Data Group collaboration, K. Nakamura et al., Review of particle physics, J. Phys. G 37 (2010) 075021 [inSPIRE].

[33] Y. Okada, M. Yamaguchi and T. Yanagida, Upper bound of the lightest Higgs boson mass in the minimal supersymmetric standard model, Prog. Theor. Phys. 85 (1991) 1 [INSPIRE].

[34] J.R. Ellis, G. Ridolfi and F. Zwirner, Radiative corrections to the masses of supersymmetric Higgs bosons, Phys. Lett. B 257 (1991) 83 [INSPIRE].

[35] H.E. Haber and R. Hempfling, Can the mass of the lightest Higgs boson of the minimal supersymmetric model be larger than $m_{Z}$ ?, Phys. Rev. Lett. 66 (1991) 1815 [INSPIRE]. 
[36] K.S. Jeong, Y. Shoji and M. Yamaguchi, Singlet-Doublet Higgs Mixing and Its Implications on the Higgs mass in the PQ-NMSSM, JHEP 09 (2012) 007 [arXiv:1205.2486] [INSPIRE].

[37] K. Nakayama, N. Yokozaki and K. Yonekura, Relaxing the Higgs mass bound in singlet extensions of the MSSM, JHEP 11 (2011) 021 [arXiv:1108.4338] [INSPIRE].

[38] A. Arvanitaki and G. Villadoro, A Non Standard Model Higgs at the LHC as a Sign of Naturalness, JHEP 02 (2012) 144 [arXiv:1112.4835] [INSPIRE].

[39] K. Blum, R.T. D'Agnolo and J. Fan, Natural SUSY Predicts: Higgs Couplings, JHEP 01 (2013) 057 [arXiv: 1206.5303] [INSPIRE].

[40] D. Carmi, A. Falkowski, E. Kuflik, T. Volansky and J. Zupan, Higgs After the Discovery: A Status Report, JHEP 10 (2012) 196 [arXiv:1207.1718] [INSPIRE].

[41] A. Djouadi, The Anatomy of electro-weak symmetry breaking. I: The Higgs boson in the standard model, Phys. Rept. 457 (2008) 1 [hep-ph/0503172] [INSPIRE].

[42] S. Heinemeyer, W. Hollik and G. Weiglein, FeynHiggs: A Program for the calculation of the masses of the neutral CP even Higgs bosons in the MSSM, Comput. Phys. Commun. 124 (2000) 76 [hep-ph/9812320] [INSPIRE].

[43] S. Heinemeyer, W. Hollik and G. Weiglein, The Masses of the neutral CP - even Higgs bosons in the MSSM: Accurate analysis at the two loop level, Eur. Phys. J. C 9 (1999) 343 [hep-ph/9812472] [INSPIRE].

[44] G. Degrassi, S. Heinemeyer, W. Hollik, P. Slavich and G. Weiglein, Towards high precision predictions for the MSSM Higgs sector, Eur. Phys. J. C 28 (2003) 133 [hep-ph/0212020] [INSPIRE].

[45] M. Frank, T. Hahn, S. Heinemeyer, W. Hollik, H. Rzehak et al., The Higgs Boson Masses and Mixings of the Complex MSSM in the Feynman-Diagrammatic Approach, JHEP 02 (2007) 047 [hep-ph/0611326] [INSPIRE].

[46] P. Gambino and M. Misiak, Quark mass effects in $\bar{B} \rightarrow X_{s} \gamma$, Nucl. Phys. B 611 (2001) 338 [hep-ph/0104034] [INSPIRE].

[47] M. Masip, R. Muñoz-Tapia and A. Pomarol, Limits on the mass of the lightest Higgs in supersymmetric models, Phys. Rev. D 57 (1998) R5340 [hep-ph/9801437] [INSPIRE].

[48] R. Barbieri, L.J. Hall, A.Y. Papaioannou, D. Pappadopulo and V.S. Rychkov, An Alternative NMSSM phenomenology with manifest perturbative unification, JHEP 03 (2008) 005 [arXiv: 0712.2903] [INSPIRE].

[49] B. Kyae and C.S. Shin, Relaxing the Landau-pole constraint in the NMSSM with the Abelian gauge symmetries, Phys. Rev. D 88 (2013) 015011 [arXiv:1212.5067] [INSPIRE].

[50] ATLAS collaboration, Search for direct production of the top squark in the all-hadronic ttbar + etmiss final state in $21 \mathrm{fb}^{-1}$ of p-pcollisions at $\sqrt{s}=8 \mathrm{TeV}$ with the ATLAS detector, ATLAS-CONF-2013-024.

[51] ATLAS collaboration, Search for direct top squark pair production in final states with one isolated lepton, jets and missing transverse momentum in sqrts $=8, T e V$ pp collisions using $21 \mathrm{fb}^{-1}$ of ATLAS data, ATLAS-CONF-2013-037.

[52] CMS Collaboration, Search for direct top squark pair production in events with a single isolated lepton, jets and missing transverse energy at $\sqrt{s}=8 \mathrm{TeV}$, CMS-PAS-SUS-12-023. 
[53] ATLAS collaboration, Updated coupling measurements of the Higgs boson with the ATLAS detector using up to $25 \mathrm{fb}^{-1}$ of proton-proton collision data, ATLAS-CONF-2014-009.

[54] ATLAS collaboration, Updated coupling measurements of the Higgs boson with the ATLAS detector using up to $25 \mathrm{fb}^{-1}$ of proton-proton collision data, ATLAS-COM-CONF-2014-013.

[55] ATLAS collaboration, Measurement of Higgs boson production in the diphoton decay channel in pp collisions at center-of-mass energies of 7 and $8 \mathrm{TeV}$ with the ATLAS detector, arXiv: 1408.7084 [INSPIRE].

[56] CMS Collaboration, Combination of standard model Higgs boson searches and measurements of the properties of the new boson with a mass near $125 \mathrm{GeV}$, CMS-PAS-HIG-13-005.

[57] CMS collaboration, Observation of the diphoton decay of the Higgs boson and measurement of its properties, Eur. Phys. J. C 74 (2014) 3076 [arXiv: 1407.0558] [INSPIRE].

[58] ATLAS collaboration, Measurements of Higgs boson production and couplings in diboson final states with the ATLAS detector at the LHC, Phys. Lett. B 726 (2013) 88 [arXiv: 1307.1427] [INSPIRE].

[59] D. Carmi, A. Falkowski, E. Kuflik, T. Volansky and J. Zupan, Higgs After the Discovery: A Status Report, JHEP 10 (2012) 196 [arXiv:1207.1718] [INSPIRE].

[60] P.M. Ferreira, J.F. Gunion, H.E. Haber and R. Santos, Probing wrong-sign Yukawa couplings at the LHC and a future linear collider, Phys. Rev. D 89 (2014) 115003 [arXiv:1403.4736] [INSPIRE].

[61] LeP Working Group for Higgs boson searches, AlePH Collaboration, Delphi Collaboration, L3 Collaboration, OPAL collaboration, R. Barate et al., Search for the standard model Higgs boson at LEP, Phys. Lett. B 565 (2003) 61 [hep-ex/0306033] [INSPIRE].

[62] LEP Higgs Working Group For Higgs boson SEARChes collaboration, Flavor independent search for hadronically decaying neutral Higgs bosons at LEP, hep-ex/0107034 [INSPIRE].

[63] K.S. Jeong, Y. Shoji and M. Yamaguchi, Peccei-Quinn invariant extension of the NMSSM, JHEP 04 (2012) 022 [arXiv: 1112.1014] [INSPIRE].

[64] J.E. Kim, H.P. Nilles and M.-S. Seo, Singlet Superfield Extension of the Minimal Supersymmetric Standard model with Peccei-Quinn symmetry and a Light Pseudoscalar Higgs Boson at the LHC, Mod. Phys. Lett. A 27 (2012) 1250166 [arXiv:1201.6547] [InSPIRE].

[65] K.J. Bae, K. Choi, E.J. Chun, S.H. Im, C.B. Park et al., Peccei-Quinn NMSSM in the light of $125 \mathrm{GeV}$ Higgs, JHEP 11 (2012) 118 [arXiv:1208.2555] [INSPIRE].

[66] LUX collaboration, D.S. Akerib et al., First results from the LUX dark matter experiment at the Sanford Underground Research Facility, Phys. Rev. Lett. 112 (2014) 091303 [arXiv: 1310.8214] [INSPIRE].

[67] T. Cohen, D.J. Phalen and A. Pierce, On the Correlation Between the Spin-Independent and Spin-Dependent Direct Detection of Dark Matter, Phys. Rev. D 81 (2010) 116001 [arXiv: 1001.3408] [INSPIRE].

[68] XENON100 collaboration, E. Aprile et al., Dark Matter Results from 225 Live Days of XENON100 Data, Phys. Rev. Lett. 109 (2012) 181301 [arXiv:1207.5988] [INSPIRE]. 
[69] P.P. Giardino, K. Kannike, I. Masina, M. Raidal and A. Strumia, The universal Higgs fit, JHEP 05 (2014) 046 [arXiv: 1303.3570] [INSPIRE].

[70] G. Bélanger, B. Dumont, U. Ellwanger, J.F. Gunion and S. Kraml, Global fit to Higgs signal strengths and couplings and implications for extended Higgs sectors, Phys. Rev. D 88 (2013) 075008 [arXiv: 1306 . 2941] [INSPIRE].

[71] OPAL collaboration, G. Abbiendi et al., Search for chargino and neutralino production at $\sqrt{s}=192 \mathrm{GeV}$ to $209 \mathrm{GeV}$ at LEP, Eur. Phys. J. C 35 (2004) 1 [hep-ex/0401026] [INSPIRE].

[72] G. Jungman, M. Kamionkowski and K. Griest, Supersymmetric dark matter, Phys. Rept. 267 (1996) 195 [hep-ph/9506380] [INSPIRE]. 